\title{
Biological Differences in Endogenous Depressive Placebo Responders versus Nonresponders: Dexamethasone Suppression Test and Sleep EEG Data
}

\author{
Gary K. Zammit, Alan H. Rosenbaum, Peter Stokes, John Davis,
} Frank Zorick, and Thomas Roth

\section{Introduction}

The association between abnormal Dexamethasone Suppression Test (DST) and sleep electroencephalographic (EEG) findings and depressives' response to active drug treatment is equivocal. Recently, however, investigators have attempted to determine the relationship between these measures and response to somatic versus nonsomatic treatment. Carroll (1985) has reported that DST nonsuppressors are not likely to respond to psychotherapy and support unless somatic treatment is also given. Similarly, Georgotas and associates (cited in Carroll 1985) found that patients with abnormal DSTs did not respond to treatment with placebo, whereas those with normal DSTs had a high rate of response. One study has even shown that abnormal DST

From the Department of Psychiatry, Eating Disorders Institute, New York Hospital-Comell Medical Center. Westchester Division, White Plains, (G.K.Z.): McAuley Hospital and the University of Michigan, Ann Arbor, MI. (A.H.R.); the Departments of Psychiatry and Medicine, New York Hospital-Cornell Medical Center, Payne Whitney Clinic, New York. NY (P.S.): the Department of Psychiatry, Illinois State Psychiatric Institute, Chicago, IL (J.D.); and the Sleep Disorders Center, Henry Ford Hospital, Detroit, MI (F.Z., T.R.).

Address reprint requests to Dr. Gary K. Zammit, Department of Psychiatry, New York Hospital-Comell Medical Center, Westchester Division, 21 Bloomingdale Road, White Plains, NY 10605. Received August 7, 1987; revised October 7. 1987. values predict a significantly poorer response to placebo than do negative ones (Peselow et al. 1986). Concurrent data have been reported for the sleep EEG. It has been shown that depressives who demonstrate both abnormal DSTs and shortened rapid eye movement (REM) sleep latencies respond poorly to cognitive psychotherapy. In contrast, patients with normal laboratory findings respond favorably to such treatment (Rush 1984).

We report data on the DST and sleep EEG in two distinct groups of patients diagnosed with endogenous depression: those that demonstrate partial or total symptom remission in response to treatment with 1 week of placebo and those who remain depressed or worsen during placebo trials. The objective is to determine if these groups, clinically similar at presentation, can be discriminated on the basis of ad hoc biological measures.

\section{Methods}

\section{Subjects}

Twenty-seven hospitalized subjects, 8 men and 19 women, participated in the study. The mean age of the group was $45.41 \pm 14.69$ years, with a range of 20-68 years. All but one of the subjects met Research Diagnostic Criteria (RDC) 
(Spitzer et al. 1978) for probable or definite major depressive disorder with endogenous features (the excepted subject was later found to meet criteria during the same episode and responded to a trial of phenelzine). In addition, all subjects had obtained a score of 20 or greatcr on the 21 -item Hamilton Depression Rating Scale (HDRS) (Hamilton 1960). All were medically healthy and drug-free.

\section{Procedure}

Upon admission to the study, subjects were interviewed using the Schedulc for Affective Disorders and Schizophrenia (SADS) (Endicott and Spitzer 1978) and the HDRS. Double-blind biological assessments were also performed on the first or second day of the study. Subjects had blood drawn for plasma cortisol at 8:00 AM, 4:00 PM, and 11:00 PM on the day of the day following a $1-\mathrm{mg}$ dose of dexamethasone at 11:00 PM. Samples wcre analyzed by radioimmunoassay procedures (described elsewhere) (Stokes et al. 1984). A postdexamethasone nonsuppression response was identified as a plasma cortisol concentration equal to or greater than $5 \mu \mathrm{g} / \mathrm{dl}$. For the purposes of our analyses. DST nonsuppression was determined separately for each time and, in addition, for all times combined (i.e., any plasma cortisol concentration of $\geqq 5$ $\mu \mathrm{g} / \mathrm{dl})$.

Sleep EEG was obtained on one night for up to $8 \mathrm{hr}$ recording time. Data were subsequently scored in accordance with the criteria of Rechtschaffen and Kales (1968). REM sleep latency was determined by computing the elapsed time occurring after sleep onset and before the first epoch of REM sleep. All intervening wakefulness was removed from this period. A REM sleep latency of $60 \mathrm{~min}$ or less was judged to be abnormally short.

On admission to the program, subjects were engaged in milieu treatment on the inpatient ward and placed on a 1-week, single-blind trial of placebo. If, at the end of that trial, subjects' HDRS scores dropped below 20 or showed a decrease of greater than or equal to $20 \%$ of their original score, they were identified as placebo responders. However, if HDRS scores remained

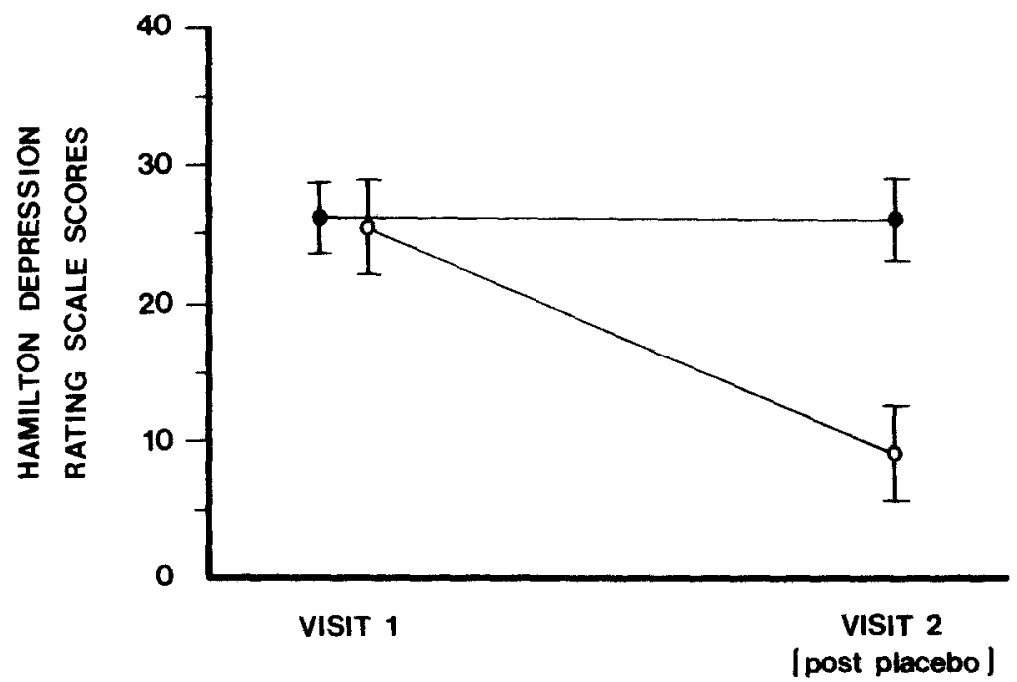

Figure 1. HDRS scores versus visit 1 and visit 2. (๑) Persistent depressives, $(0)$ placebo responders. 
Table 1. Sleep Parameters: Means \pm SDs Between Groups

\begin{tabular}{lccc}
\hline \multicolumn{1}{c}{ Variable $^{a}$} & Placebo responders & Persistent depressives & $t$ \\
\hline Time in bed & $488.43 \pm 18.92$ & $480.09 \pm 28.57$ & 0.70 \\
Total sleep time & $417.07 \pm 44.56$ & $363.66 \pm 52.96$ & $2.32^{c}$ \\
Wake before sleep & $21.79 \pm 15.10$ & $50.56 \pm 51.43$ & 1.44 \\
Wake during sleep & & $55.31 \pm 47.25$ & 0.61 \\
Wake after sleep & $42.93 \pm 38.25$ & $9.94 \pm 17.30$ & 0.48 \\
Number of wakes & $6.64 \pm 7.84$ & $5.25 \pm 2.89$ & 1.41 \\
Total min wake & $7.86 \pm 6.09$ & $115.81 \pm 50.29$ & 1.95 \\
Percent wake & $71.36 \pm 50.57$ & $24.10 \pm 10.28$ & $2.08^{c}$ \\
Minutes Stage 1 & $14.47 \pm 10.04$ & $49.19 \pm 26.41$ & 1.26 \\
Minutes Stage 2 & $66.50 \pm 38.34$ & $209.28 \pm 53.97$ & 1.02 \\
Minutes Stage 3/4 & $233.86 \pm 51.66$ & $26.78 \pm 30.91$ & 0.22 \\
Minutes REM & $23.86 \pm 26.91$ & $78.50 \pm 30.91$ & 1.14 \\
Movement time & $92.64 \pm 15.32$ & $0.50 \pm 1.03$ & 0.68 \\
Latency Stage 1 & $0.21 \pm 0.57$ & $25.63 \pm 20.27$ & 0.82 \\
Latency Stage 2 & $18.50 \pm 16.43$ & $50.34 \pm 49.80$ & 1.20 \\
Latency Stage REM & $27.14 \pm 14.93$ & $60.03 \pm 23.68$ & $1.99^{d}$ \\
Percent Stage 1 & $85.64 \pm 37.72$ & $13.78 \pm 7.55$ & 0.60 \\
Percent Stage 2 & $15.91 \pm 8.63$ & $57.37 \pm 12.86$ & 0.22 \\
Percent Stage 3/4 & $56.14 \pm 11.40$ & $7.33 \pm 8.25$ & 0.45 \\
Percent REM & $5.74 \pm 6.27$ & $21.40 \pm 7.04$ & 0.26 \\
\hline
\end{tabular}

${ }^{a}$ In minutes, unless otherwise noted.

${ }^{b}$ Wake after sleep onset but before final awakening.

${ }^{c} p<0.05$.

${ }^{d} p<0.06$.

stable, increased, or showed a moderate $(<20 \%)$ decline, subjects were identified as persistent depressives (or placebo nonresponders). Using these criteria, even some small declines in HDRS scores were judged as indices of response, despite the fact that these may not have constituted clinical remission.

\section{Results}

Using the clinical criteria cited, seven subjects were identified as placebo responders, and 20 were identified as persistent depressives. No age or sex difference was observed between groups. Analyses of HDRS scores obtained on admission and after 1 week of placebo are summarized in Figure 1. No difference in severity of depression was seen on admission, but placebo responders show an appreciable decline in HDRS scores $(t=7.89$, $p<0.001$ ), as compared to persistent depressives whose scores essentially remained the same $(t=0.17$, NS). The duration of depressive episodes, although somewhat greater in the placebo responders, was no different between groups $(t=1.70$, NS).

$T$-tests were used to determine differences in EEG measures of sleep (Table 1). The results show that persistent depressives have significantly less total sleep time and greater percent wake time than placebo responders. Abnormal REM latency was observed in 8 of the $16(50 \%)$ persistent depressives and 1 (14\%) placebo responder. This trend approaches significance (Fisher-Irwin Exact, $p=0.12$ ). Furthermore, when latency to REM is expressed as an absolute value (in minutes), there is a near-significant difference between groups (see Table 1).

DST results show that there are no be- 
tween-groups differences in predexamethasone plasma cortisol levels obtained at any time or in postdexamethasone plasma cortisol levels at 8:00 AM and 11:00 PM. At 4:00 PM, however, a significant difference was observed between the two groups $\left(\chi^{2}=7.80, p<0.01\right)$. Thirteen of $20(65 \%)$ persistent depressives displayed positive DST results, whereas none of the placebo responders had an abnormal result (see Figure 2). When defining nonsuppression as any postdexamethasone cortisol value equal to or greater than $5 \mu \mathrm{g} / \mathrm{dl}$, a significant difference is also observed, with persistent depressives demonstrating a greater occurrence of abnormal results $\left(\chi^{2}=4.34, p<0.05\right)$.

When both biological dependent variables were viewed in combination, only 3 of $16(19 \%)$ persistent depressives assessed had both an abnormal REM latency and DST at 8:00 AM, 6 of $16(38 \%)$ at $4: 00 \mathrm{PM}$, and 6 of 15 at 11:00 PM $(40 \%)$. However, 12 of $16(75 \%)$ had either an abnormal REM latency or onc abnormal DST result. This was significantly different from placebo responders $\left(\chi^{2}=4.41, p<0.05\right)$, as only two had either test abnormal.

\section{Discussion}

The patients in this study presented with diagnoses of unipolar endogenous depression and initially appeared to be a clinically homogeneous sample when rated using the SADS, RDC, and HDRS. When the sample is divided into groups of placebo responders and nonresponders (persistent depressives), differences in the DST and sleep EEG can be seen. Persistent depressives, as a group, tend to have a greater number of abnormal DST results at 4:00 PM, less total sleep time, and greater percent wake time than do placebo responders. Nonsignificant trends also suggest that shortened REM latency is more frequently observed in persistent depressives than in placebo responders. Finally, a significantly greater proportion of persistent depressives have at least one biological measure (the DST or REM latency) that is abnormal. These data are meaningful in that they suggest that biological abnormalities in depression may not simply be associated with symptom presentation and that the DST and sleep EEG may predict response or nonresponse to placebo.

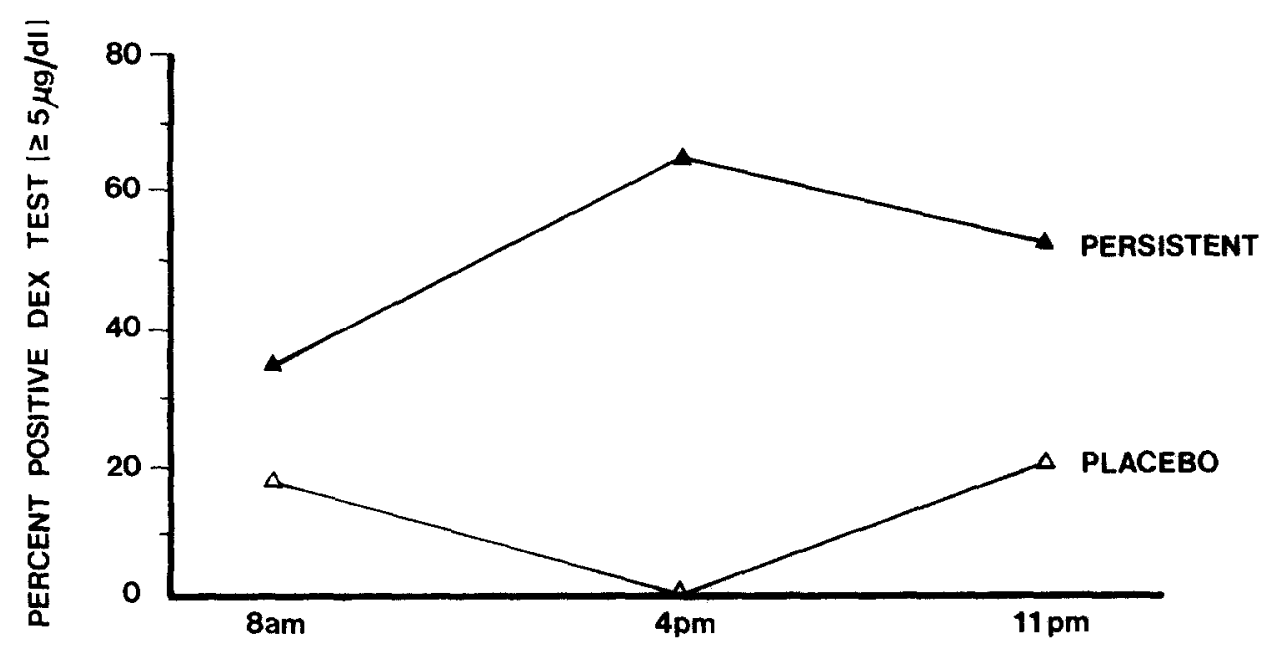

TIME

Figure 2. Postdexamethasone plasma cortisol. 
The findings reported here must be considered preliminary. They are limited by the small sample size, the lack of double-blind placebo administration, and the absence of follow-up (to determine if placebo responders subsequently relapse). Additional well-controlled studies are needed to determine if biological measures may be developed as clinically useful ad hoc indices of treatment response.

The authors would like to thank Dr. Charles Pollak for reviewing drafts of the manuscript. This work was supported in part by NIMH grant RO $1 \mathrm{MH}-38677$ and a grant from the Hexter foundation (Dr. Stokes), and by PHS award/grant IT32MH183890-01A1 (Dr. Zammit).

\section{References}

Carroll BJ (1985): Dexamethasone Suppression Test: A review of contemporary confusion. J Clin Psychiatry 46:13-24.

Endicott J, Spitzer RL (1978): A diagnostic inter- view: The Schedule for Affective Disorders and Schizophrenia. Arch Gen Psychiatry 35:837-844.

Hamilton M (1960): A rating scale for depression. $J$ Neurol Neurosurg Psychiatry 23:56-62.

Peselow ED, Lautin A, Wolkin A, Rohrs C, Novatt A, Siekierski J, Rotrosen J (1986): The Dexamethasone Suppression Test and response to placebo. J Clin Psychopharmacol 6:286-291.

Rechtschaffen A, Kales A (1968): A Manual of Standardized Terminology, Techniques and Scoring System for Sleep Stages of Human Subjects. Washington DC: US Government Printing Office (DHHS Publication No. 204).

Rush AJ (1984): A Phase 2 study of cognitive therapy of depression. In Williams JBW, Spitzer RL (eds), Psychotherapy Research: Where Are We and Where Should We Go? New York: Guilford Press.

Spitzer RL, Endicott J, Robins E (1978): Research Diagnostic Criteria. Arch Gen Psychiatry 35:773782.

Stokes PE, Stoll PM, Koslow SH, Maas JW, Davis JM, Swann AC, Robins E (1984): Pretreatment DST and hypothalamic-pituitary-adrenocortical function in depressed patients and comparison groups. Arch Gen Psychiatry 41:257-267. 